\title{
ON MATRIX CONVEXITY OF THE MOORE-PENROSE INVERSE
}

\section{B. MOND}

Department of Mathematics

La Trobe University

Bundoora, Victoria, 3083, AUSTRALIA

\section{J.E. PEČARIĆ}

Faculty of Textil Technology

University of Zagreb

Zagreb, CROATIA

(Received December 12, 1994 and in revised form June 28, 1995)

\begin{abstract}
Matrix convexity of the Moore-Penrose inverse was considered in the recent literature Here we give some converse inequalities as well as further generalizations
\end{abstract}

KEY WORDS AND PHRASES: Matrix convexity, generalized inverse 1991 AMS SUBJECT CLASSIFICATION CODES: 15A45, 15 A09.

\section{INTRODUCTION}

Let $A$ and $B$ be two complex Hermitian positive definite matrices, and let $0 \leq \lambda \leq 1$ Then

$$
[\lambda A+(1-\lambda) B]^{-1} \leq \lambda A^{-1}+(1-\lambda) B^{-1}
$$

where $A \geq B$ means that $A-B$ is a positive semi-definite matrix.

This result, i e., matrix convexity of the inverse function is an old result that appears explicitly in the papers $[1,2,3,4,5]$ (see also the books [6, pp 554-555] and [7, pp. 469-471]).

The related matrix convexity of the Moore-Penrose (generalized) inverse, denoted by $A^{+}$, was considered in paper $[8,9,10]$ The following was given in [10]:

Let $A$ and $B$ be two complex Hermitian positive semi-definite matrices of the same order. The inequality

$$
[\lambda A+(1-\lambda) B]^{+} \leq \lambda A^{+}+(1-\lambda) B^{+}
$$

for every $0 \leq \lambda \leq 1$ holds if and only if

$$
R(A)=R(B)
$$

where $R(A)$ is the range of $A$.

Two converses of (1.1) were obtained in [11]:

If $A$ and $B$ are complex Hermitian positive definite matrices and $0 \leq \lambda \leq 1$ is a real number, then

$$
[\lambda A+(1-\lambda) B]^{-1} \geq K\left(\lambda A^{-1}+(1-\lambda) B^{-1}\right)
$$

and

where

$$
[\lambda A+(1-\lambda) B]^{-1}-\left(\lambda A^{-1}+(1-\lambda) B^{-1}\right) \geq \tilde{K} A^{-1}
$$

$$
K=4 \min _{2} \frac{\mu_{\imath}}{\left(1+\mu_{2}\right)^{2}}, \quad \tilde{K}=\min _{2} \frac{\left(\sqrt{\mu_{2}}-1\right)^{2}}{-\mu_{2}},
$$

and the $\mu_{2}$ are the solutions of the equation

$$
\operatorname{det}(B-\mu A)=0 \text {. }
$$

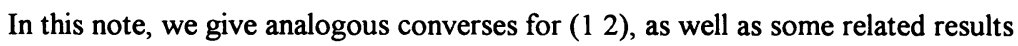

\section{CONVERSES OF THE MATRIX CONVEXITY INEQUALITY OF THE MOORE-PENROSE INVERSE}

Let $A$ and $B$ be two complex Hermitian positive semi-definite matrices of the same order such that (13) holds Let $P$ be a unitary matrix such that $A=P \operatorname{diag}\left(A_{1}, 0\right) P^{*}$ where $A_{1}$ is a diagonal positive definite matrix When (1 3 ) holds, we have $B=P \operatorname{diag}\left(B_{1}, 0\right) P^{*}$ where $B_{1}$ is positive definite 
THEOREM 1. Let $A$ and $B$ be two complex Hermitian positive semi-definite matrices of the same order such that ( 13 ) holds and let $0 \leq \lambda \leq 1$ Then

$$
[\lambda A+(1-\lambda) B]^{+} \geq K\left(\lambda A^{+}+(1-\lambda) B^{+}\right)
$$

where $K$ is defined by ( $16 \mathrm{a}$ ) and the $\mu_{\imath}$ are the positive solutions of the equation

$$
\operatorname{det}\left(B_{1}-\lambda A_{1}\right)=0 \text {. }
$$

THEOREM 2. Let $A, B$ be defined as in Theorem 1 Then

$$
\left[\lambda A+(1-\lambda) B^{+}\right]-\left(\lambda A^{+}+(1-\lambda) B^{+}\right) \geq \tilde{K} A^{+}
$$

where $\tilde{K}$ is defined by ( $16 \mathrm{~b}$ ) and the $\mu_{2}$ are positive solutions of the equation (2 2)

PROOF. By (1 4) and (1 5) we have

$$
\left[\lambda A_{1}+(1-\lambda) B_{1}\right]^{-1} \geq K\left(\lambda A_{1}^{-1}+(1-\lambda) B_{1}^{-1}\right)
$$

and

$$
\left[\lambda A_{1}+(1-\lambda) B_{1}\right]^{-1}-\left(\lambda A_{1}^{-1}+(1-\lambda) B_{1}^{-1}\right) \geq \tilde{K} A_{1}^{-1}
$$

where $K$ is defined by (16a), $\tilde{K}$ by $(16 \mathrm{~b})$ and the $\mu_{2}$ are solutions of $(22)$ Since $P A^{+} P^{*}=\left(P A P^{*}\right)^{+},(2.1)$ follows from (24) and (2 3) from (2.5)

\section{SOME RELATED RESULTS}

Let $(Y, B, \mu)$ be a probability space and $A_{y}, y \in Y$ a collection of positive semi-definite matrices of the same order. Let $A_{y}=\left(a_{\imath y y}\right), 1 \leq i, j \leq n$ and $y \in Y$ Assume that $a_{\imath \jmath y}$ as a function of $y$ is measurable for every $1 \leq i, j \leq n$ The following results were proved in $[9,10]$

Suppose there exists a set $D \in B$ such that $\mu(D)=1$ and $A_{y 1} A_{y 2}=A_{y 2} A_{y 1}$ for every $y_{1}, y_{2} \in D$ Let $R\left(A_{y}\right)$ be the same for all $y \in D \in B$. Suppose $A_{y}$ and $A_{y}^{+}$as functions of $y$ are integrable with respect to $\mu$ Then

$$
\left[\int_{Y} A_{y} \mu(d y)\right]^{+} \leq \int_{Y} A_{y}^{+} \mu(d y) .
$$

By $\int_{Y} A_{y} \mu(d y)$ we mean the matrix whose $(i, j)^{t h}$ element is $\int_{Y} a_{\imath y y} \mu(d y)$

THEOREM 3. If also all positive eigenvalues of $A_{y}$ for all $y \in Y$ are in the interval $[m, M]$ where $0<m<M$, then the following inequalities hold

$$
\int_{Y} A_{y}^{+} \mu(d y) \leq \frac{(M+m)^{2}}{4 M m}\left[\int_{Y} A_{y} \mu(d y)\right]^{+}
$$

and

$$
\int_{Y} A_{y}^{+} \mu(d y)-\left[\int_{Y} A_{y} \mu(d y)\right]^{+} \leq \frac{(\sqrt{M}-\sqrt{m})^{2}}{M m} I .
$$

PROOF. As in [9], we have that there exists an orthogonal matrix $C$ such that

$$
C^{T} A C=\operatorname{diag}\left\{\lambda_{\imath y}, \lambda_{2 y}, \ldots, \lambda_{n y}\right\}, \quad y \in Y
$$

where $\lambda_{1 y}, \lambda_{2 y}, \ldots, \lambda_{n y}$ are the eigenvalues of $A_{y}$ Since $A_{y}$ is positive semi-definite, each $\lambda_{\imath y} \geq 0$. Let $k$ be the rank of $A_{y}$ We can assume without loss of generality that

$\lambda_{1 y}, \lambda_{2 y}, \ldots, \lambda_{k y} \neq 0$ for every $y \in Y, \quad$ and $\quad \lambda_{k+1, y}=\lambda_{k+2, y}=\ldots \lambda_{n y}=0$ for every $y \in Y$.

Note that

$$
A_{y}^{+}=C \operatorname{diag}\left\{\frac{1}{\lambda_{1 y}}, \frac{1}{\lambda_{2 y}}, \ldots, \frac{1}{\lambda_{k y}}, 0, \ldots, 0\right\} C^{T}
$$

so that 
ON MATRIX CONVEXITY OF THE MOORI:-PENROSE INVERSE

709

$$
C^{\prime} A_{y}^{\prime} C=\operatorname{diag}\left\{\frac{1}{\lambda_{1 y}}, \frac{1}{\lambda_{2 y}}, \ldots, \frac{1}{\lambda_{k y}}, 0, \ldots, 0\right\} .
$$

Thus, we have

$$
\begin{aligned}
& K\left[\int_{Y} A_{y} \mu(d y)\right]^{\prime}-\int_{Y} \lambda_{y}^{+} \mu(d y)=C \operatorname{diag}\left\{K\left(\int_{Y} \lambda_{1 y} \mu(d y)\right)^{-1}\right. \\
& \left.\quad-\int_{Y} \lambda_{1 y}^{1} \mu d y, \ldots, K\left(\int_{Y} \lambda_{k y} \mu(d y)\right)^{1}-\int_{Y} \lambda_{k y}^{-1} \mu(d y), 0, \ldots, 0\right\} C^{T}
\end{aligned}
$$

where $K=(M+m)^{2} /(4 M m)$ The inequality

$$
K\left[\int_{Y} \lambda_{\imath y} \mu(d y)\right]^{-1} \int_{Y} \lambda_{\imath y}^{-1} \mu(d y)
$$

is the well-known Kantorovich inequality Hence each diagonal element in the above diagonal matrix is nonnegative This completes the proof of (3 2)

Similarly,

$$
\begin{aligned}
\int_{Y} A_{y}^{+} \mu(d y)-\left[\int_{Y} A_{y} \mu(d y)\right]^{+}-\tilde{K} I & =C \operatorname{diag}\left\{\int_{Y} \lambda_{1 y}^{-1} \mu(d y)-\left(\int_{Y} \lambda_{1 y} \mu(d y)\right)^{-1}\right. \\
& \left.\quad-\tilde{K}, \ldots, \int \lambda_{k y}^{-1} \mu(d y)-\left(\int_{Y} \lambda_{k y} \mu(d y)\right)^{-1}-\tilde{K},-\tilde{K}, \ldots,-\tilde{K}\right\} C^{T}
\end{aligned}
$$

where $\tilde{K}=\frac{(\sqrt{M}-\sqrt{m})^{2}}{M m}$ The inequality

$$
\int_{Y} \lambda_{\imath y}^{-1} \mu(d y)-\int_{Y} \lambda_{\imath y} \mu(d y)^{-1} \leq \tilde{K}
$$

is a simple consequence of the following Mond-Shisha inequality [12]

$$
\int f-\left(\int f^{-1}\right)^{-1} \leq(\sqrt{M}-\sqrt{m})^{2}
$$

where $m \leq f \leq M, 0<m<M$. Namely

$$
\begin{gathered}
\frac{1}{M} \leq \frac{1}{f} \leq \frac{1}{m} \quad \text { so that by substituting } f \rightarrow \frac{1}{f}, \text { we get } \\
\int f^{-1}-\left(\int f\right)^{-1} \leq \frac{(\sqrt{M}-\sqrt{m})^{2}}{M m}=\tilde{K} .
\end{gathered}
$$

Thus each diagonal element in the above diagonal matrix is non-positive. This completes the proof

Moreover, we can consider the powers of $A$ and $A^{+}$. For simplicity of notation, if $r<0$, we shall use $A^{(r)}$ for $\left(A^{+}\right)^{-r}$. Note that $\left(A^{+}\right)^{-r}=\left(A^{-r}\right)^{+}$

THEOREM 4. Let $R\left(A_{y}\right)$ be the same for all $y \in D \in B$. Suppose $A_{y}^{s}$ and $A_{y}^{(r)},(r<0<s)$ as functions of $y$ are integrable with respect to $\mu$ Then

$$
\left[\int_{Y} A_{y}^{(r)} \mu(d y)\right]^{s} \geq\left[\int_{Y} A_{y}^{s} \mu(d y)\right]^{(r)}
$$

PROOF. As in the proof of (3.2) and (3 3), we have

$$
\begin{aligned}
{\left[\int_{Y} A_{y}^{(r)} \mu(d y)\right]^{s}-\left[\int_{Y} A_{y}^{s} \mu(d y)\right]^{(r)}=} & C \operatorname{diag}\left\{\left(\int_{Y} \lambda_{1 y}^{r} \mu(d y)\right)^{s}-\left(\int_{Y} \lambda_{1 y}^{s} \mu(d y)\right)^{r}, \ldots\right. \\
& \left.\left(\int_{Y} \lambda_{k}^{r} \mu(d y)\right)^{s}-\left(\int_{Y} \lambda_{k y}^{s} \mu(d y)\right)^{r}, 0, \ldots, 0\right\} C^{T} .
\end{aligned}
$$


Each diagonal element in the above diagonal matrix is nonnegative This follows from the fact that if $f^{s}$ and $f^{r}$ are positive and integrable, the well-known inequality for means of orders $s$ and $r$ states that

$$
\left(\int f^{r}\right)^{1 / r} \leq\left(\int f^{\prime}\right)^{1 / 9} \quad(r<0<s)
$$

which is the same as

$$
\left(\int f^{s}\right)^{r} \leq\left(\int f^{r}\right)^{s}
$$

Similar consequences of converse inequalities for (3 5) (see [12] and [13], respectively) are the next two theorems

THEOREM 5. Let the conditions of Theorem 4 be satisfied and let all positive eigenvalues of $A_{y}$ for all $y \in Y$ belong to the interval $[m, M](0<m<M)$ Then the following inequality holds

$$
\left[\int_{Y} A_{y}^{s} \mu(d y)\right]^{(r)} \geq \triangle\left[\int_{Y} A_{y}^{(r)} \mu(d y)\right]^{s}
$$

where

$$
\Delta=\left\{\frac{r\left(\gamma^{s}-\gamma^{r}\right)}{(s-r)\left(\gamma^{r}-1\right)}\right\}^{r}\left\{\frac{s\left(\gamma^{r}-\gamma^{s}\right)}{(r-s)\left(\gamma^{s}-1\right)}\right\}^{-s}, \quad \gamma=M / m
$$

THEOREM 6. Let the conditions of Theorem 5 be satisfied Then

$$
\left[\int_{Y} A_{y}^{(r)} \mu(d y)\right]^{s}-\left[\int_{y} A_{y}^{s} \mu(d y)\right]^{(r)} \leq \Lambda I
$$

where

$$
\Lambda=\max _{\theta \in[0,1]}\left\{\left[\theta M^{r}+(1-\theta) m^{r}\right]^{s}-\left[\theta M^{s}+(1-\theta) m^{s}\right]^{r}\right\}
$$

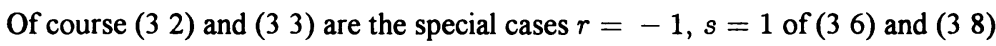

\section{REFERENCES}

[1] BENDAT, J and SHERMAN, S., Monotone and convex operator functions, Trans. Amer. Math. Soc. 79 (1955), 58-71

[2] DAVIS, C, Notions generalizing convexity for functions defined on spaces of matrices, Proc. Symp. Pure Math., Vol 7 Convexity, Amer. Math. Soc (1963), 187-201

[3] MOORE, M H, A convex matrix function, Amer. Math. Monthly 80 (1973), 408-409

[4] OLKIN, I. and PRATT, J, A multivariate Tchebycheff inequality, Ann. Math. Statist. 29 (1958), 226-234

[5] WHITTLE, P, A multivariate generalization of Tchebychev's inequality, Quart. J. Math. Oxford, Ser [2] 9 (1958), 232-240

[6] HORN, R.A. and JOHNSON, C.R., Topics in Matrix Analysis, Cambridge University Press, New York, 1991.

[7] MARSHALL, A.W. and OLKIN, I., Inequalities: Theory of Majorization and its Applications, Academic Press, New York, 1979.

[8] GIOVAGNOLI, A. and WYNN, H.P., G-majorization with application to matrix orderings, Lin. Alg. Appl. 67 (1985), 111-135

[9] KAFFES, D G., An inequality for matrices, Bull. Greek Math. Soc. 22 (1981), 143-159

[10] KAFFES, D G, MATHEW, T., RAO, M B and SUBRAMANYAM, K, On the matrix convexity of the Moore-Penrose inverse and some applications, Lin. Multilin. Alg. 24 (1989), 265-271

[11] MOND, B and PEČARIĆ, J E., Reverse forms of a convex matrix inequality, Lin. Alg. Appl., to appear

[12] MOND, B and SHISHA, O, Difference and ratio inequalities in Hilbert space, in Inequalltes, Vol 2, Academic Press, New York, 1970, 241-249

[13] PEČARIĆ, J E and BEESACK, P R., On Knopp's inequality for convex functions, Canad. Math. Bull. 30 (1987), 267-272 


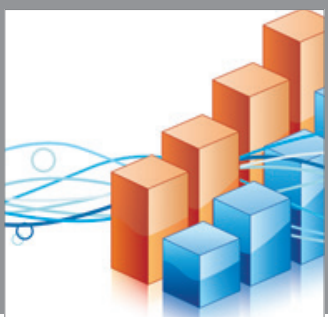

Advances in

Operations Research

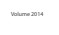

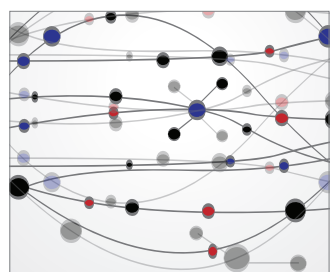

\section{The Scientific} World Journal
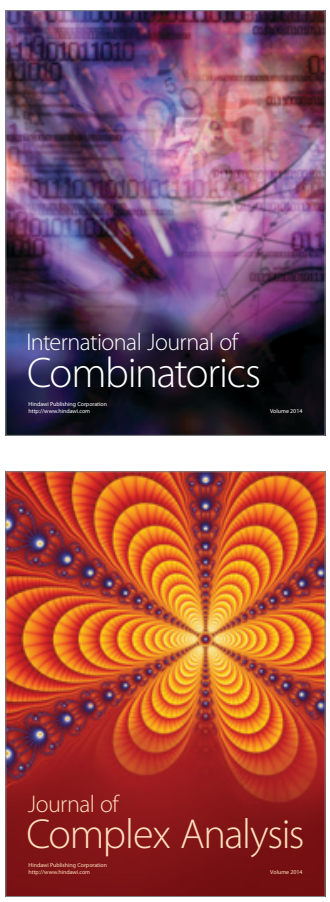

International Journal of

Mathematics and

Mathematical

Sciences
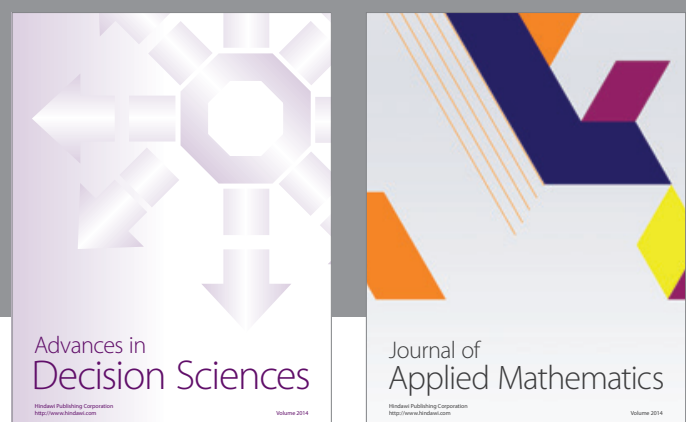

Journal of

Applied Mathematics
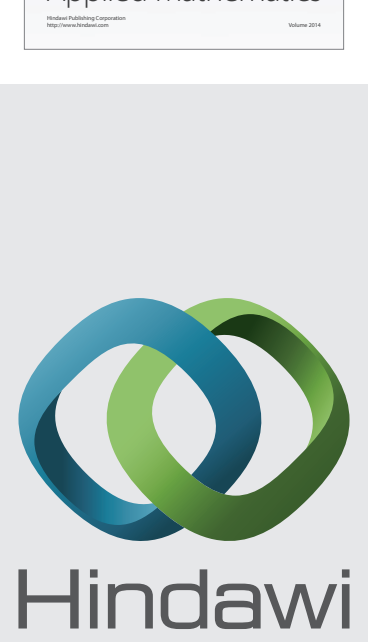

Submit your manuscripts at http://www.hindawi.com
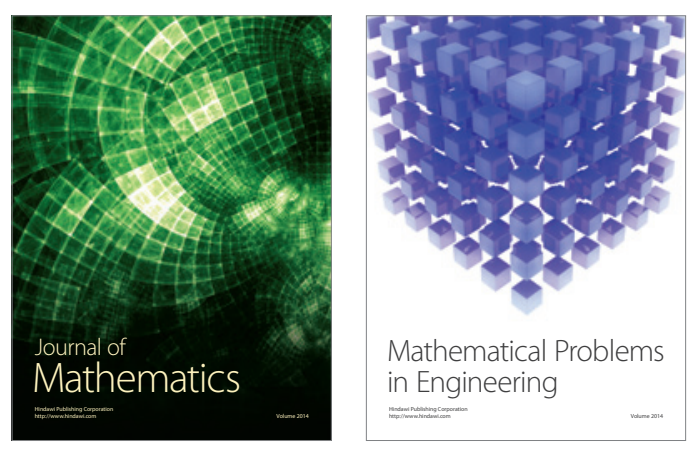

Mathematical Problems in Engineering
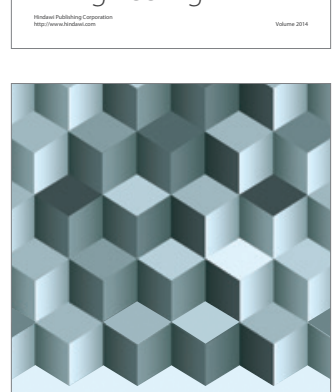

Journal of

Function Spaces
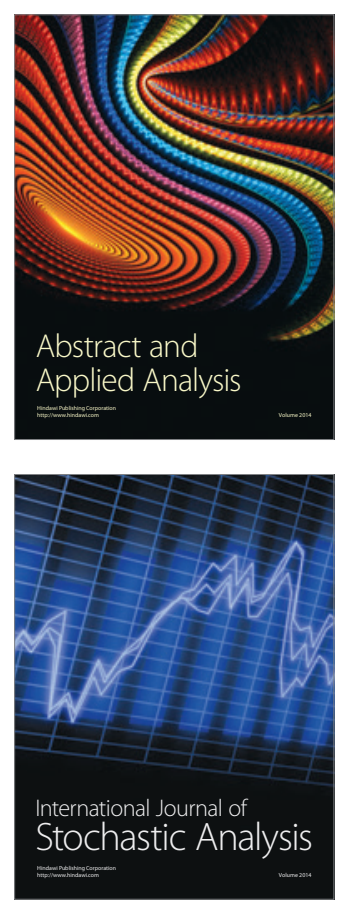

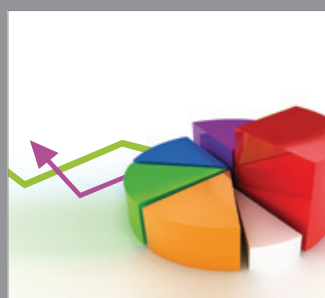

ournal of

Probability and Statistics

Promensencen
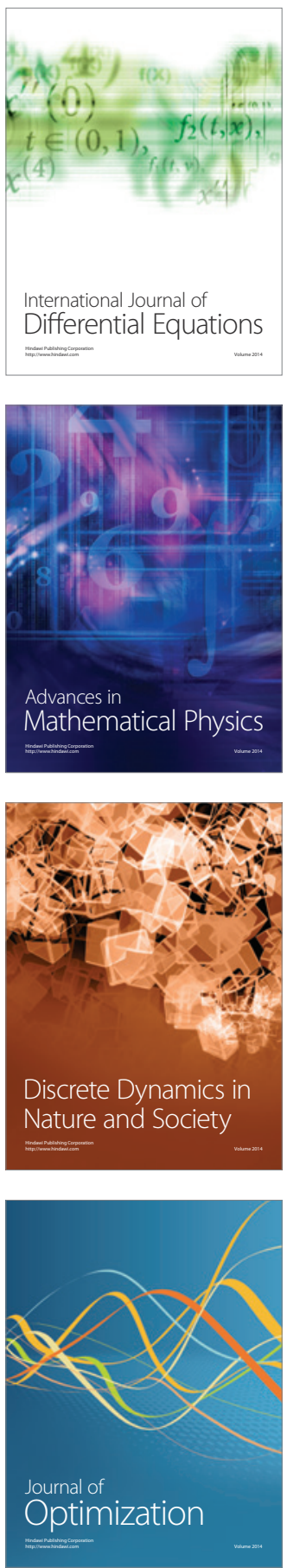